\title{
Sexually active groups in cattle-A novel estrus sign
}

\author{
G. Sveberg, ${ }^{*} \dagger^{1}$ A. O. Refsdal, $\dagger$ H. W. Erhard, $¥ \S$ E. Kommisrud,\# M. Aldrin,II I. F. Tvete,II F. Buckley,ף \\ A. Waldmann, ${ }^{* *}$ and E. Ropstad* \\ *Department of Production Animal Clinical Sciences, Norwegian School of Veterinary Science, N-0033 Oslo, Norway \\ †GENO Breeding and AI Association, N-2317 Hamar, Norway \\ $\ddagger$ AgroParisTech, UMR791 Modélisation Systémique Appliquée aux Ruminants, 75005 Paris, France \\ §INRA, UMR791 Modélisation Systémique Appliquée aux Ruminants, 75005 Paris, France \\ \#Faculty of Education and Natural Sciences, Hedmark University College, N-2418 Elverum, Norway \\ IINorwegian Computing Center, N-0373 Oslo, Norway \\ TAnimal and Bioscience Research Department, Animal and Grassland Research and Innovation Centre, Teagasc, \\ Dairy Production Research Centre, Moorepark, Fermoy, Co. Cork, Ireland \\ ${ }^{* *}$ Department of Reproductive Biology, Institute of Veterinary Medicine and Animal Sciences, Estonian University of Life Sciences, 51014 Tartu, \\ Estonia
}

\section{ABSTRACT}

The current study presents a novel objective measure for characterizing sexually active groups (SAG 3-5) and relates this measure to other behaviors of lactating Holstein-Friesian cows. Cows in SAG 3-5 were required to participate in a minimum of 1 estrus behavior per $5 \mathrm{~min}$ while staying within $3 \mathrm{~m}$ (2 cow lengths) of its partner(s) for a minimum of $5 \mathrm{~min}$. Twenty Holstein-Friesian cows were video-monitored continuously through 1 complete estrous cycle (22 d). Standing behavior, SAG 3-5, secondary estrus signs (SEC), and other social and agonistic behaviors were recorded continuously. The period of mounting estrus (MTE) was divided into the 3 parts: prestand, standing estrus (STE), and poststand. The mean durations of MTE, prestand, STE, and poststand period were $12.9 \pm 1.84,4.0 \pm 1.93,7.1 \pm 1.44$, and $1.8 \pm 0.57 \mathrm{~h}(\mathrm{n}=13)$. The fractions of time spent in SAG 3-5 during MTE, prestand, STE, and poststand period were $13,8,19$, and $1 \%(\mathrm{n}=11)$. During MTE, cows participated, on average, in 5.8 \pm 1.24 SAG 3-5 and initiated $9.5 \pm 2.99$ mounts, with mean durations of $0.25 \pm 0.03 \mathrm{~h}$ and $4.00 \pm 0.36 \mathrm{~s}$, respectively. The novel measure SAG 3-5 was a sign of long duration not confined only to groups of STE cows. On one day when no cows were in estrus and during the periods 4 to $24 \mathrm{~h}$ before and after MTE, no SAG 3-5 behaviors were observed. Luteal-phase cows participated in SAG 3-5 only when the partner was a single cow in estrus. The time spent in SAG 3-5 increased between 1 and $3 \mathrm{~h}$ before MTE and the prestand period (3 vs. 8\%) and reached a peak level during STE. From STE to poststand, time spent in SAG 3-5 decreased consider-

Received November 22, 2012.

Accepted March 16, 2013.

${ }^{1}$ Corresponding author: guro.sveberg@tine.no ably (19 vs. 1\%). The observed decrease in nonmutual agonistic behaviors 4 to $24 \mathrm{~h}$ before MTE is suggested as an early sign of pre-estrus. Changes in SAG 3-5, agonistic behaviors, and SEC are suggested as indicators of the specific stages of MTE. Increased SEC initiated and SAG 3-5 were indicators of late pre-estrus and early estrus (prestand). Peak levels of SAG 3-5, SEC, and social agonistic behaviors were indicators of STE. A sudden decrease in behaviors, preceded by frequent interactions, was indicative of late estrus (poststand). On the basis of the findings reported here, we propose that SAG 3-5, as well as proceptive and receptive patterns of SEC and agonistic behaviors, be included in estrus detection protocols. Updated knowledge of these behavioral interactions may assist when determining the stage of estrus and the optimal time to breed dairy cows.

Key words: sexually active group (SAG 3-5), agonistic behavior, mounting estrus, standing estrus

\section{INTRODUCTION}

Detection of estrus is one of the major challenges in dairy cattle management. This task is challenged by the reported shortening of the estrus period and fewer cows expressing primary estrus signs over the past 30 to $50 \mathrm{yr}$ (Dobson et al., 2008). Updated knowledge of estrus symptoms and reliable detection methods are essential for improving the reproductive outcome in dairy herds (Coleman, 1993; Van Vliet and van Eerdenburg, 1996). A stronger focus on other behaviors associated with estrus has been suggested to sustain and improve heat detection (Kilgour et al., 1977; Kerbrat and Disenhaus, 2004). The specific stages in mounting estrus (MTE), standing estrus (STE), and the periods before and after STE are described by Hurnik et al. (1975). However, few studies report changes in behaviors as- 
sociated with these periods. Kerbrat and Disenhaus (2004) and Sveberg et al. (2011) described social and sexual behaviors around estrus. Although reporting that changes in sexual behavior are the best indicators of the estrus period, Kerbrat and Disenhaus (2004) confirmed that mounting only occurred during estrus, in agreement with the original definitions of estrus as being the mount period (Hurnik et al., 1975). Sveberg et al. (2011) based their study on STE (true estrus) and revealed specific behavioral changes the hours before and after STE. The start and end of both STE and MTE are associated with the time of ovulation (Roelofs et al., 2005). All behavioral changes that can identify specific stages in estrus; that is, start and end of MTE and start and end of STE, should be identified and described, as mounting and standing behaviors are often absent in estrous cows (Van Vliet and van Eerdenburg, 1996; Orihuela, 2000). Identifying signs and understanding behavioral patterns associated with specific stages of estrus may improve estrus detection and decisions about the most appropriate time to breed dairy cattle.

Sexual activity is traditionally recorded as single events. Primary and secondary estrus signs are of short duration (Hurnik, 1987) and might be difficult to use in heat detection (Dobson et al., 2008). Other social behaviors are known to be associated with estrus (Kerbrat and Disenhaus, 2004; Sveberg et al., 2011) and to influence the manifestation and detection of estrus (Hafez and Lindsay, 1965; Galina et al., 1996). Intense female-female interactions, expressed by estrous cows staying close to each other in sexually active groups (SAG), are described in the literature as characteristic of cattle as a species (Hafez and Bouissou, 1975; Albright and Arave, 1997). This tendency of estrous cows to approach and remain in the vicinity of other cows, even when other signs of estrus are not expressed, is probably known among farmers. Despite this, information on how these interactions can be used in estrus detection is not commonly available in the scientific literature.

To our knowledge, all previous studies identified SAG by recording groups of cows participating in mounting or standing to be mounted (Kilgour et al., 1977; Ramírez-Iglesia et al., 2006; Law et al., 2009). RamírezIglesia et al. (2006) suggested that the detection of SAG could contribute to improving the efficiency of estrus detection in cattle. The longer durations of SAG reported in their study imply that SAG could be easier to discover than single estrus signs of reported short durations (Hurnik et al., 1975).

To our knowledge, no reports define and quantify SAG, other than those based on the number of cows in STE. A previous report (Sveberg et al. 2011), describ- ing behaviors through a complete estrous cycle by continuous video surveillance, revealed that estrous cows tended to stand close to other cows while performing mutual estrus behavior. The objective of the present study was to develop measures to describe the SAG in a qualitative and quantitative manner and to relate these measures to other behaviors through complete estrous cycles of lactating Holstein-Friesian cows.

\section{MATERIALS AND METHODS}

All procedures were carried out under experimental license issued by the Irish Department of Health and Children, in accordance with the European Communities (Amendment of Cruelty to Animals Act 1876) Regulations 2005.

\section{Animals, Housing, and Management}

Twenty Holstein-Friesian cows were housed on an outdoor pad from April 11 to May 16, 2006, at the Teagasc Moorepark Ballydague research farm in Ireland. The study duration was $22 \mathrm{~d}$, beginning on April 18. The pad was $17 \times 24 \mathrm{~m}$, with wood chip flooring (O'Driscoll et al., 2008). Cows were offered freshly harvested grass ad libitum twice daily, $2.6 \mathrm{~kg}$ of concentrate DM per cow in the milking parlor, and unlimited access to fresh water. Cows were in first to sixth parity, with a frequency distribution of $8,3,2,4,2$, and 1 , respectively. Cows included in the study were required to be at least 5 wk postpartum, to have no abnormal signs in the reproductive tract upon examination by ultrasound, lameness scores of $\leq 2$ (Sprecher et al., 1997), and BCS of at least 2.5 (Lowman et al., 1976).

The cows were milked twice daily (0700 and 1530 h), using a parallel milking stall with $2 \times 20$ milking places. Individual cow milk yield was recorded using electronic milk meters (Dairymaster, Causeway, Co. Kerry, Ireland). Reproductive organs were examined by ultrasound (Aloka SSD-500, Tokyo, Japan), equipped with a $5-\mathrm{MHz}$ rectal transducer, between d 28 and 35 and d 50 and 60 postpartum to reveal reproductive disorders and evidence for ovarian cyclicity. Body condition score was scored monthly postpartum and $14 \mathrm{~d}$ before the start of the study. Body weights were recorded weekly.

\section{Ovarian Function}

Daily whole milk samples were drawn from each cow to measure progesterone concentrations. A potassium dichromate preservative tablet (Lactab Mark III, Thompson \& Capper Ltd., Cheshire, UK) was immediately added to daily samples, which were then cooled, 
labeled, and frozen within $2 \mathrm{~h}$ at $-20^{\circ} \mathrm{C}$ until measurement of progesterone concentrations by enzyme immunoassay (Waldmann, 1993), modified by using the second antibody coating technique. The specificity of the monoclonal antibody 9C11 is described by Waldmann (1999). The interassay coefficients of variation for milk progesterone concentrations of 1.48 and $19.66 \mathrm{ng} / \mathrm{mL}$ were 9.21 and $5.32 \%$, respectively. The intraassay coefficient of variation was $<10 \%$. The sensitivity limit, using a $20-\mu \mathrm{L}$ milk sample, was $<0.5 \mathrm{ng} / \mathrm{mL}$. Function of the corpus luteum (cyclicity) was confirmed by progesterone profiling, characterized by successive waves of increasing and decreasing progesterone values. Events of STE were confirmed by concurrent observation of low milk progesterone concentrations $(<2.5 \mathrm{ng} / \mathrm{mL})$ during these periods.

\section{Definitions Used for Stages of the Estrous Cycle and Behavior}

The definitions by Hurnik et al. (1975) were modified and used as follows. Standing estrus (STE; true estrus) was defined as the interval between the first and last standing events, at which the cow makes no effort to escape when mounted by other cows. This interval may coincide with, or be shorter than, the mounting period.

Mounting estrus (MTE; estrus) was defined as the interval from the first to the last mount engaged in by the cow going through STE, or cyclic nonstanding cows with confirmed low progesterone concentrations during this period. At least 2 mounts within $24 \mathrm{~h}$ were required.

Prestand was defined as the period between the start of the MTE and the start of the STE; poststand was defined as the period between the end of the STE and the end of the MTE. Pre-estrus was defined as the 24-h period before MTE; post-estrus was defined as the 24-h period after MTE.

The SAG behavior in this study (SAG 3-5) was defined by the following criteria: cow participating in a minimum of 1 estrus behavior per 5 min with its sexually active partner(s) while staying within $3 \mathrm{~m}(2$ cow lengths; Kondo et al., 2003; Sveberg et al., 2007) of the partner(s) for at least $5 \mathrm{~min}$. The duration of SAG 3-5 started with the first sexual behavior initiated or received (mount, attempt to mount, anogenital sniff, or chin rest) and continued until a period of 5 min without sexual behavior had elapsed or until the cow left the group or was left by its SAG 3-5 partner(s).

Additional behavioral definitions included the following: (1) primary estrus sign: cow remains stationary when mounted; the cow makes no effort to escape when mounted by other cows; (2) secondary estrus signs (SEC): mount, attempt to mount, anogenital sniff, and chin rest; (3) agonistic behaviors: body butt, push away, chase up, chase away, threat, winner, and avoid (Mülleder et al., 2003; Sveberg et al., 2011). Head lean, social lick, and rump lick were regarded as other individual social behaviors (Mülleder et al., 2003; Sveberg et al., 2011). Mutual agonistic social behaviors (Mülleder et al., 2003; Sveberg et al., 2011) were head butt and play rub (sum of head play and head rub). It was not possible to differentiate between initiator and receiver of these mutual agonistic behaviors.

All identified SAG 3-5 events were reconfirmed after the initial video analysis, to identify equivalent times for partner cows joining and leaving the SAG 3-5 cow. One cow could join or leave the existing SAG 3-5 alone only when 2 or more cows already participated in the current SAG 3-5. The minimum duration of 5 min was chosen for practical reasons; this was considered the minimum time window required to observe and detect estrus signs in a group of cattle. Videos were analyzed retrospectively and the resulting data were entered into a database (Sveberg et al., 2011). To avoid investigator bias, the researcher analyzing the video recordings had no knowledge of the estrous cycle stage of the individual animals.

Estrus signs, agonistic and other social behaviors, and SAG 3-5 were recorded throughout the study period. For an event to be defined as a standing event the mounted cow had to stand still and the mounting had to last for a minimum of $2 \mathrm{~s}$ (2 consecutive video pictures). Standing when mounted sideways or head-wise was also considered to be a standing event. All first and last standing events, and the respective preceding and following mounts, were confirmed by 1 additional video observer.

Cows were observed continuously through 1 estrous cycle for a period of at least $21 \mathrm{~d}$, until all cows had shown either a regular cyclic change in progesterone concentrations and STE when progesterone was $<1$ $\mathrm{ng} / \mathrm{mL}$, or only a regular cyclic progesterone profile without STE. Behavioral observations were continued for $24 \mathrm{~h}$ after the last observation where the final cow expressing STE stood to be mounted, resulting in a study period of $22 \mathrm{~d}$ of all cows. Records from $1 \mathrm{~d}$ on which no cows expressed STE, including the preceding and following days and all cows had progesterone levels of $\geq 2 \mathrm{ng} / \mathrm{mL}$, were used for within-cow comparisons of STE and nonestrous behavior. The luteal phase was defined as the period between d 7 and 16 after the end of STE. For those cows whose study period was completed during the luteal phase, data from the previous estrous cycle, up to $4 \mathrm{~d}$ before estrus, were added. Thereby, data from an estimated luteal phase of 10-d duration was obtained for each cow. The periods $24 \mathrm{~h}$ before and after MTE, MTE, prestand, STE, poststand, the $1 \mathrm{~d}$ 
of nonestrus, and the luteal phase of the estrous cycle were analyzed because the data did not reveal major changes in other phases of the cycle. The periods $24 \mathrm{~h}$ before and after the MTE period were further divided into shorter 3-h intervals (Roelofs et al., 2005; Sveberg et al., 2011).

\section{Behavior Recording and Data Management}

Two cameras (Axis 211, Axis Communications, Lund, Sweden), both covering the area of the outdoor pad, were placed $3.5 \mathrm{~m}$ above the ground at both corners of one of the long sides of the rectangular pad. Individual identification codes were painted on the neck, flank, and upper leg of each cow to facilitate identification. To allow continuous video surveillance, artificial white light was used at night.

\section{Statistical Analyses}

Associations between numbers of cows standing, milk yield, and parity (independent variables), and SAG 3-5 fraction were analyzed using multiple linear regression, with SAG 3-5 as the dependent variable. The effect of parity on secondary estrus behaviors and social behaviors initiated or received was analyzed, using linear regression, with parity (primiparous vs. multiparous) as the independent variable.

The mean frequency (counts per hour) of behavioral signs, mean fraction (\%) of the period expressing SAG 3-5 behavior, and mean duration of SAG 3-5 were calculated for periods of the estrous cycle defined previously, and comparisons between periods were made for the cows with at least 2 standing events during MTE. The mean frequencies were calculated as the total number of each behavior during the period divided by the total length of the period, where total means the sum over all cows with at least 2 standing events. This can be expressed as $\Sigma_{i} x_{i j} / \Sigma_{i} t_{i j}$, where $x_{i j}$ is the number of a specific behavior for the $i$ th cow in the $j$ th period, and $t_{i j}$ is the corresponding length of the $j$ th period (Sveberg et al., 2011). The mean fractions during the specific time periods were calculated similarly, as the time spent in SAG 3-5 during a period divided by the total length of the same period. This can be expressed by the same formula as above, but $x_{i j}$ represents the time in SAG 3-5 for the $i$ th cow in period $j$. The mean durations of SAG 3-5 in MTE were calculated from the SAG 3-5 starting and ending during MTE. Mean duration of SAG 3-5 in STE period were similar to the respective SAG 3-5 durations of MTE period and therefore only SAG 3-5 duration of the MTE period were estimated.
The period-wise differences between the mean fraction of SAG 3-5 and an adjusted mean frequency of SEC initiated were calculated as SAG3-5 ${ }_{j}^{\text {frac }}-c \cdot$ SECinit ${ }_{j}^{\text {frac }}$, where $S A G 3-5_{j}^{\text {frac }}$ is the mean SAG 3-5 fraction, SECinit frac is the mean SEC frequency in period $j$, and $c$ is an adjustment factor so that the average of $S A G 3-5_{j}^{\text {frac }}$ over all periods is the same as the average of $c \cdot S E C i n i t_{j}^{f r a c}$, and the average of the period-wise differences is zero. The period-wise differences between the mean fraction of SAG 3-5 and an adjusted mean frequency of received secondary estrus signs were calculated the same way, but with a different adjustment factor.

The data consisted of repeated measurements on each cow, where observations within each cow were correlated, and simple formulas for the standard error of a mean could not be used to calculate estimation uncertainties. Therefore, the uncertainties of the frequency and fraction estimates were calculated by the bootstrap procedure (Efron and Tibshirani, 1993; Sveberg et al., 2011), taking into account the repeated measurement design of the data.

\section{RESULTS}

The 20 cows included in the study were, on average, $63 \mathrm{~d}$ postpartum (range 44 to $84 \mathrm{~d}$ ). The 15 cows in MTE had a 305-d mean milk yield of 6,073 \pm 305.2 $( \pm \mathrm{SE}) \mathrm{kg}$. Their mean BCS was $2.95 \pm 0.061$, BCS change was $0.00 \pm 0.049$, and mean BW change was $13.11 \pm 2.314 \mathrm{~kg}$ during the study period. Based on progesterone measurements, 16 of 20 cows were characterized as cyclic. All cyclic cows expressed MTE, and 14 out of 16 MTE cows expressed STE. Twelve of the 16 cows that expressed MTE participated in SAG 3-5, and they all expressed STE. One of the 16 cyclic cows was excluded because the duration of her estrous cycle was $25 \mathrm{~d}$ and consequently observations for a full estrus cycle were incomplete. The 2 cows expressing MTE but no STE had only 1 mount during MTE. In addition, 2 of 13 cows in STE had only 1 standing event and other signs could therefore not be described during STE for these 4 cows.

\section{Duration of Estrus and Estrus Behaviors}

An average of $5.8 \pm 1.24$ SAG 3-5 with a mean duration of the single SAG $3-5$ of $0.25 \pm 0.03 \mathrm{~h}$ were observed during MTE. Cows in STE $(7.1 \pm 5.19 \mathrm{~h}$; $\mathrm{n}=13$ ) participated in SAG 3-5 for $19 \%$ of the time (Table 1). The duration of MTE and the time spent in SAG 3-5 during MTE was $12.9 \pm 6.64 \mathrm{~h}$ and $13 \%$, 
Table 1. Comparison of sexually active group (SAG 3-5) fraction ${ }^{1}$ (in \%) between periods ${ }^{2}$ of estrous cycle

\begin{tabular}{|c|c|c|c|c|c|c|c|c|}
\hline Period $1^{3}$ & Period $2^{3}$ & \multicolumn{3}{|c|}{ Period 1} & \multicolumn{3}{|c|}{ Period 2} & $P$-value ${ }^{4}$ \\
\hline NOE & MTE & 0 & 0 & 0 & 13 & 8 & 19 & $<0.001$ \\
\hline Pre-MTE13-24 & Pre-MTE10-12 & 0 & 0 & 0 & 0 & 0 & 0 & 0.72 \\
\hline Pre-MTE10-12 & Pre-MTE7-9 & 0 & 0 & 0 & 0 & 0 & 0 & $\mathrm{NA}^{6}$ \\
\hline Pre-MTE7-9 & Pre-MTE4-6 & 0 & 0 & 0 & 0 & 0 & 1 & 0.70 \\
\hline STE & Post-STE & 19 & 11 & 26 & 1 & 0 & 3 & $<0.001$ \\
\hline Post-STE & Post-MTE1-3 & 1 & 0 & 3 & 1 & 0 & 2 & 0.60 \\
\hline Post-MTE1-3 & Post-MTE4-6 & 1 & 0 & 2 & 0 & 0 & 0 & 0.23 \\
\hline Post-MTE4-6 & Post-MTE7-9 & 0 & 0 & 0 & 0 & 0 & 0 & NA \\
\hline Post-MTE7-9 & Post-MTE10-12 & 0 & 0 & 0 & 0 & 0 & 0 & NA \\
\hline Post-MTE10-12 & Post-MTE13-24 & 0 & 0 & 0 & 0 & 0 & 0 & NA \\
\hline
\end{tabular}

${ }^{1}$ Fraction $=$ time spent in SAG 3-5 out of total time spent in period; SAG 3-5 = event defined by cow participating in a minimum of 1 estrus behavior per 5 min with its sexually active partner(s) while staying within $3 \mathrm{~m}$ (2 cow lengths).

${ }^{2}$ Period 1 and 2 of compared periods.

${ }^{3} \mathrm{NOE}=$ selected day with no cows in estrus; pre-MTE $\mathrm{x}-\mathrm{y}=$ time before mount estrus (MTE), where $\mathrm{x}$ and $\mathrm{y}$ indicate the hour interval; pre-/ post-STE $=$ before/after standing estrus (STE); post-MTE $\mathrm{x}-\mathrm{y}=$ time after mount estrus (MTE), where $\mathrm{x}$ and $\mathrm{y}$ indicate the hour interval.

${ }^{4} P$-value for the test of difference between period 1 and 2 .

${ }^{5}$ Lower/Upper $=$ lower/upper limit of $95 \%$ CI of period.

${ }^{6} \mathrm{NA}=$ not available.

respectively, for the 13 cows expressing STE. During prestand $(4.0 \pm 6.94 \mathrm{~h})$, the STE cows participated in SAG 3-5 for $8 \%$ of the time, and during poststand (1.8 $\pm 2.06 \mathrm{~h}$ ), the STE cows participated in SAG 3-5 for $1 \%$ of the time. The mean duration of MTE for all cows that expressed it $(\mathrm{n}=15)$ was $11.1 \pm 7.63 \mathrm{~h}$. During MTE, on average, $9.5 \pm 2.99$ mounts were initiated, with a mean duration of $4.00 \pm 0.36 \mathrm{~s}$.

\section{Sexually Active Group Fraction}

Table 1 and Figure 1a describe SAG 3-5 fractions through different stages of the estrous cycle. The differences in SAG 3-5 fraction between periods in estrus were most prominent for nonestrus versus MTE period, 1 to $3 \mathrm{~h}$ before MTE versus prestand, and STE versus poststand period (Table 1; Figure 1). Only 1 cow participated in SAG 3-5 during the poststand period, and this SAG 3-5 was observed in the first hour after STE.

\section{Effect of Number of Cows in STE}

Cows in MTE and STE participated in 55 (93\%) and $46(78 \%)$ out of the total of 59 observed SAG 3-5 events, respectively. During periods with at least one cow in STE, the estrous cow participated in all observed SAG 3-5. Seven out of the 13 STE cows were always alone in STE, 5 cows were partly alone and partly simultaneously with other cow(s) in STE, and 1 cow was always simultaneously with other STE cows during STE. Dur- ing periods of 2 cows simultaneously in SAG 3-5, the estrous cow spent, on average, 3.6 times more time in SAG 3-5 compared with periods of cow alone in STE $(36 \pm 6.4 \%$ vs. $10 \pm 3.0 \%)$. Of all cows that acted as partners when a single cow was SAG 3-5, $43 \%$ were in the luteal phase, $32 \%$ were in the prestand period, $11 \%$ were in the period 1 to $7 \mathrm{~d}$ before or after MTE, $7 \%$ were in the period 1 to $24 \mathrm{~h}$ before MTE, $3.5 \%$ were in the poststand period, and $3.5 \%$ were acyclic.

\section{Nonestrous Cows}

Seven out of the 15 cows (47\%) expressing MTE participated in at least 1 SAG 3-5 event during their luteal stage, and $15(27 \%)$ of the total of 55 SAG 3-5 events observed during MTE included luteal cows. Ten (67\%) of 15 SAG 3-5 partners were in STE, 3 partners (20\%) were in prestand, 1 partner $(7 \%)$ was in poststand, and 1 SAG 3-5 partner (7\%) of luteal SAG 3-5 cows was in the period $1 \mathrm{~h}$ before MTE. Two cows, $1 \mathrm{~h}$ before and 6 $\mathrm{h}$ after MTE, respectively, participated as a third cow in these groups. Only 1 of 4 acyclic cows participated in $1 \mathrm{SAG} 3-5$ during the 22-d period, as a partner to a cow alone in STE.

\section{Secondary Estrus Signs}

Figure $2 \mathrm{~b}$ describes SEC through the respective stages of estrous cycle. The difference between SEC initiated versus received was significant during the periods 4 to 6 $\mathrm{h}$ before MTE, 1 to $3 \mathrm{~h}$ before MTE, and prestand, but 

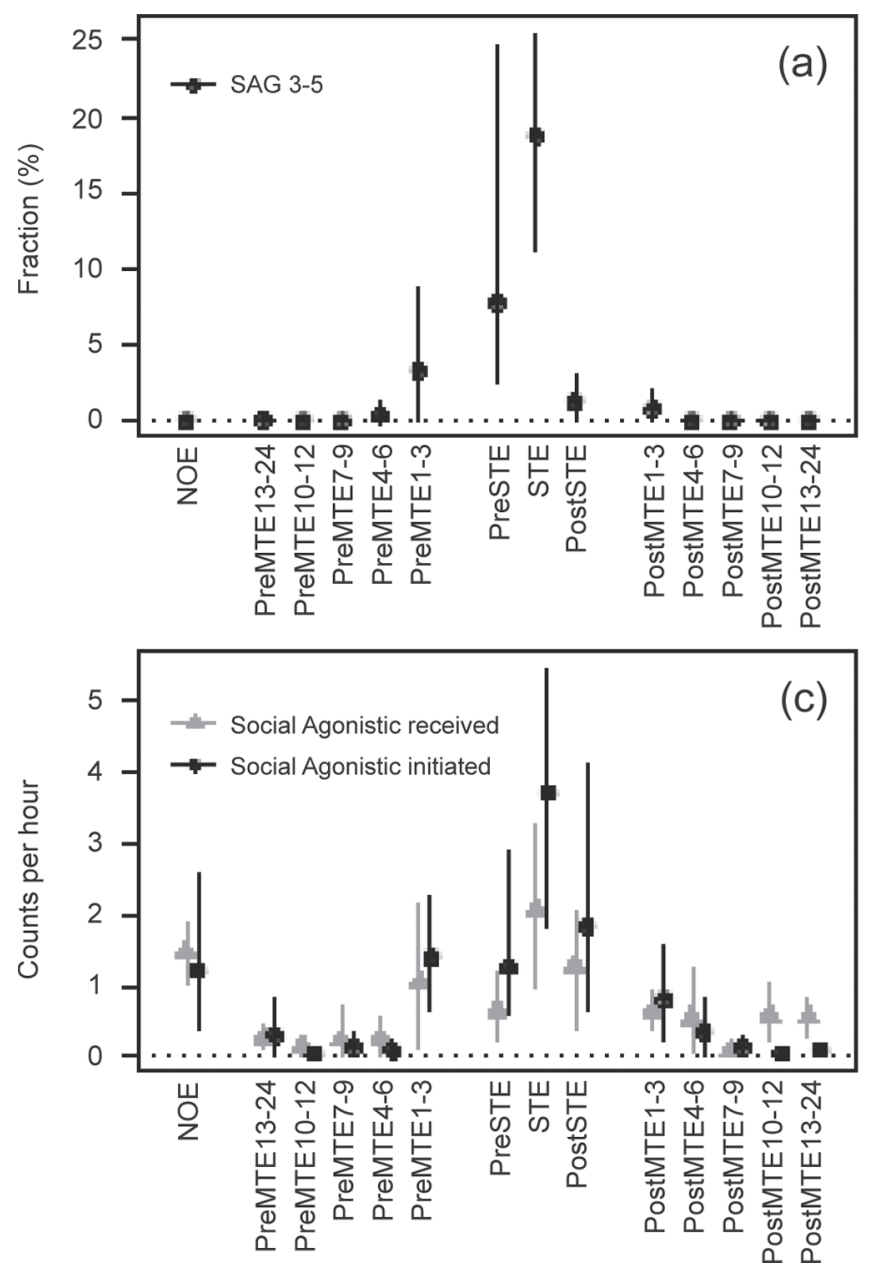
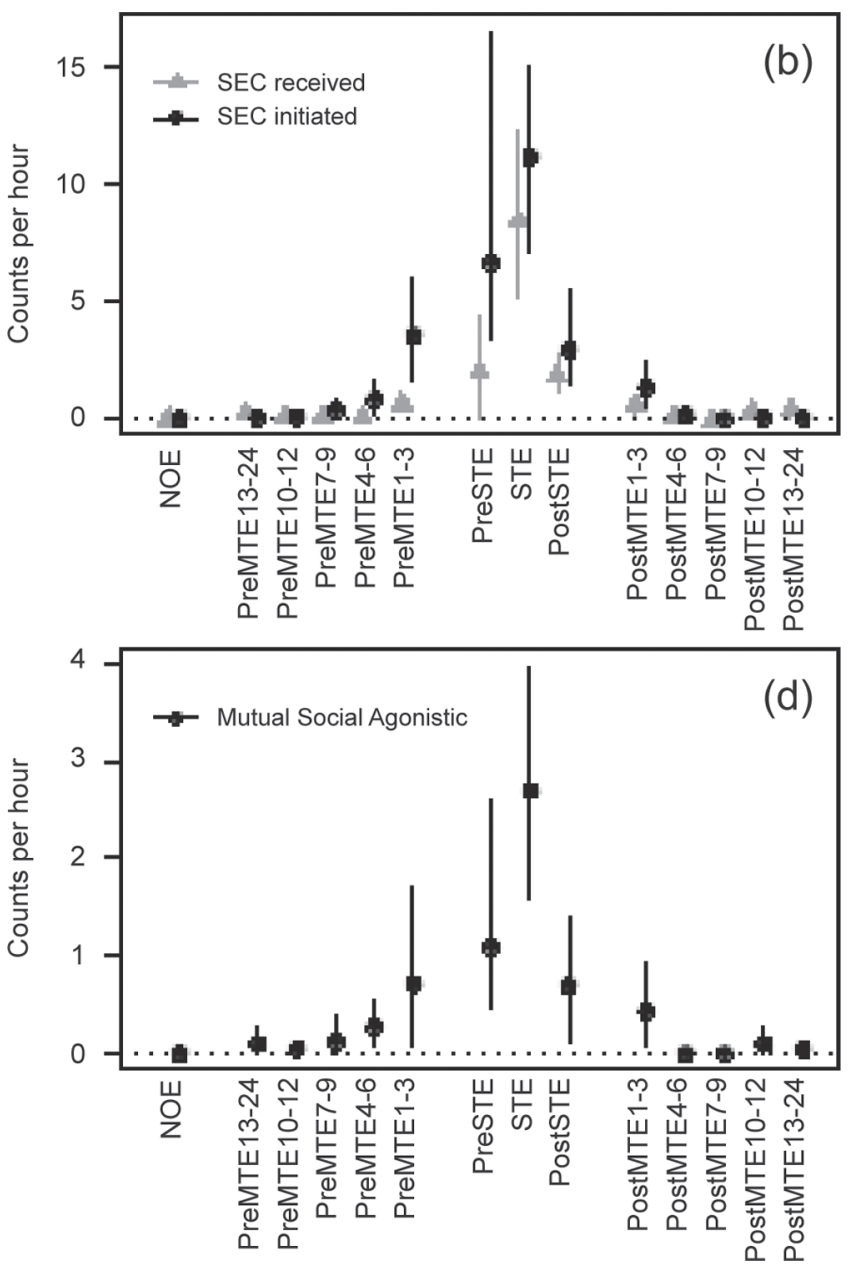

Figure 1. Estimates of expected (a) fractions (time spent in sexually active group, SAG 3-5, out of total time spent in period, measured in $\%$ ), (b) frequencies of SEC (secondary estrus signs: anogenital sniff, chin rest, attempt to mount and sum of mounts) initiated (•) and received $(\boldsymbol{\Lambda}),(\mathrm{c})$ frequencies of social (head lean, social lick, rump lick) and agonistic (body butt, push away, chase up, chase away, threat, winner, avoid) behavior initiated $(\mathbf{)})$ and received $(\boldsymbol{\Lambda})$, and (d) frequencies of mutual social agonistic behavior (head butt, play rub), with $95 \%$ confidence intervals at specific stages of estrous cycle. NOE = selected day with no cows in estrus; pre-MTE $\mathrm{x}-\mathrm{y}=$ time before mount estrus (MTE), where $\mathrm{x}$ and y indicate the hour interval; pre-/post-STE = before/after standing estrus (STE); post-MTE x-y = time after mount estrus (MTE), where $\mathrm{x}$ and $\mathrm{y}$ indicate the hour interval. SAG $3-5=$ event defined by cow participating in a minimum of 1 estrus behavior per 5 min with its sexually active partner(s) while staying within $3 \mathrm{~m}$ (2 cow lengths).

not during STE (Table 2). Receptive behavior (SEC received) changed most prominently from prestand to STE and from STE to poststand. Proceptive behavior (SEC initiated) changed most prominently from STE to poststand (Table 2).

\section{Differences Between SAG and SEC}

The fraction of SAG 3-5 and the frequency of SEC both increased considerably during STE. Figure 2 shows that the difference between SAG 3-5 fraction and the frequency of adjusted SEC initiated behaviors became increasingly negative in the periods from 12 to $3 \mathrm{~h}$ before pre-STE, whereas the difference between SAG 3-5 fraction and frequency of adjusted SEC received behav- iors became increasingly positive. The most prominent positive and negative changes were found during STE and post-STE, respectively. The difference between SAG 3-5 fraction and frequency of adjusted SEC initiated behaviors decreased gradually from post-STE during the period 1 to $24 \mathrm{~h}$ after MTE. The difference between SAG 3-5 and adjusted SEC received followed a different pattern; the difference was increasing from 4 to $6 \mathrm{~h}$ before MTE, the most prominent positive and negative differences were found during prestand and poststand, respectively, and no differences were found during STE. From post-STE to $9 \mathrm{~h}$ after MTE, the difference between SAG 3-5 and adjusted SEC received followed similar patterns as of SAG 3-5 and adjusted SEC initiated. 
Table 2. Comparison between secondary estrus signs (SEC) initiated and received (measured as counts per hour) at specific stages of estrous cycle

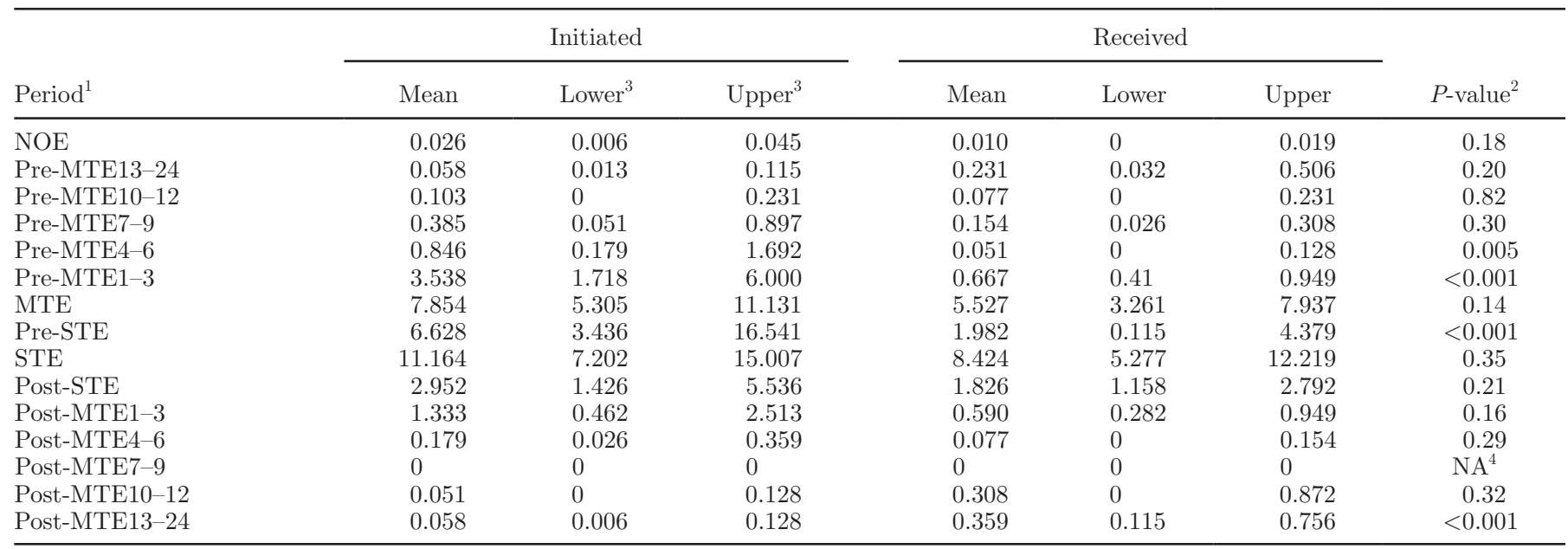

${ }^{1} \mathrm{NOE}=$ selected day with no cows in estrus; pre-MTE $\mathrm{x}-\mathrm{y}=$ time before mount estrus (MTE), where $\mathrm{x}$ and $\mathrm{y}$ indicate the hour interval; pre- $/$ post-STE $=$ before/after standing estrus (STE); post-MTE $\mathrm{x}-\mathrm{y}=$ time after mount estrus (MTE), where $\mathrm{x}$ and $\mathrm{y}$ indicate the hour interval.

${ }^{2} P$-value for the test of difference between SEC behaviors initiated and received.

${ }^{3}$ Lower/Upper $=$ lower/upper limit of $95 \%$ CI of period.

${ }^{4} \mathrm{NA}=$ not available.

\section{Agonistic Behavior}

The highest frequencies of other agonistic behavior were observed during STE, but in contrast to SEC and mutual agonistic behaviors, high frequencies were also found in nonestrus and followed by low frequencies 6 to $24 \mathrm{~h}$ before MTE (Figure 1c). Mutual agonistic behaviors (head butt and play rub) followed patterns similar to SEC through the estrous cycle; a gradual increase was observed 7 to $9 \mathrm{~h}$ before MTE, followed by high frequencies during STE and a decrease after STE (Figure 1d). More social agonistic behaviors were initiated than were received during STE (Table 3), in contrast to SEC behaviors, which were initiated and received to a similar extent during STE (Table 2). Differences in mutual agonistic behaviors were observed

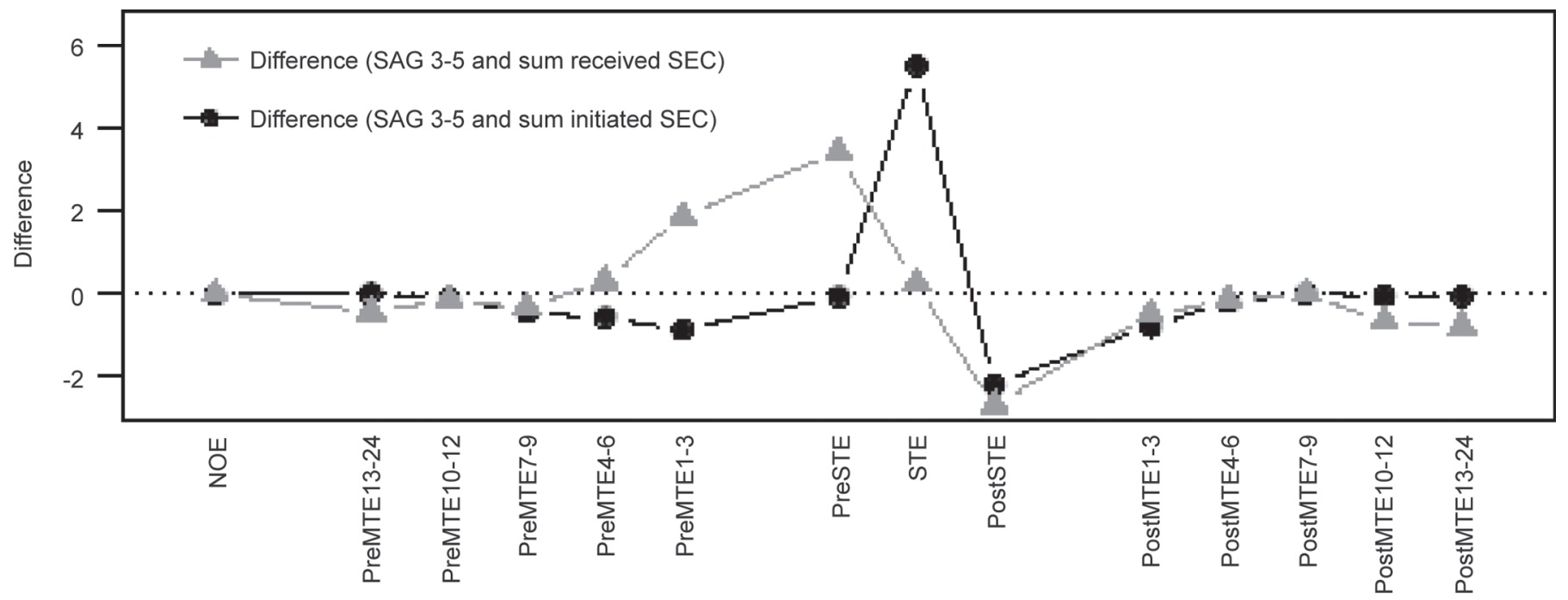

Figure 2. Differences between the mean fraction (\%) of sexually active groups (SAG 3-5) and adjusted mean frequency of secondary estrus

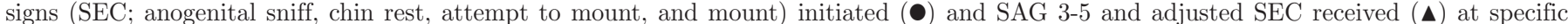
stages of estrous cycle. $\mathrm{NOE}=$ selected day with no cows in estrus; pre-MTE $\mathrm{x}-\mathrm{y}=$ time before mount estrus (MTE), where $\mathrm{x}$ and $\mathrm{y}$ indicate the hour interval; pre-/post-STE = before/after standing estrus (STE); post-MTE $\mathrm{x}-\mathrm{y}=$ time after mount estrus (MTE), where $\mathrm{x}$ and y indicate the hour interval. SAG 3-5 = event defined by cow participating in a minimum of 1 estrus behavior per 5 min with its sexually active partner(s) while staying within $3 \mathrm{~m}$ ( 2 cow lengths). 
Table 3. Comparison between agonistic behavior initiated and received (measured as counts per hour) through periods of estrous cycle

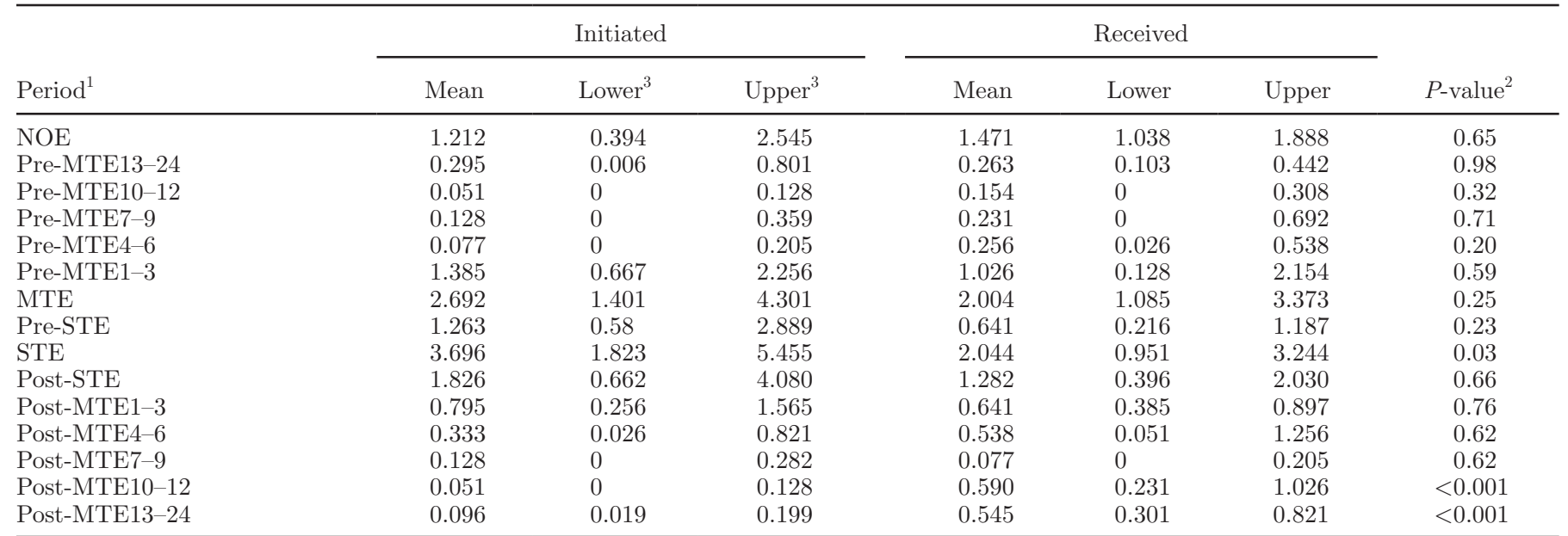

${ }^{1} \mathrm{NOE}=$ selected day with no cows in estrus; pre-MTE $\mathrm{x}-\mathrm{y}=$ time before mount estrus (MTE), where $\mathrm{x}$ and $\mathrm{y}$ indicate the hour interval; pre-/ post-STE $=$ before/after standing estrus (STE); post-MTE $\mathrm{x}-\mathrm{y}=$ time after mount estrus (MTE), where $\mathrm{x}$ and $\mathrm{y}$ indicate the hour interval.

${ }^{2} P$-value for the test of difference between agonistic behaviors initiated and received.

${ }^{3}$ Lower/Upper $=$ lower/upper limit of $95 \%$ CI of period.

between nonestrus and MTE, 7 to $9 \mathrm{~h}$ versus 4 to $6 \mathrm{~h}$ before MTE, STE versus post-stand, and 1-3 h versus 4-6 h after MTE (Table 4).

\section{DISCUSSION}

\section{Defining Estrus and SAG 3-5}

The present study describes a measure characterizing and quantifying sexually active groups (SAG 3-5) and relates SAG 3-5 and agonistic and sexual behaviors to specific stages of estrous cycle. Standing estrus is described as the period of choice to most successfully breed cattle (Trimberger, 1948). The MTE period may be a better practical definition of estrus (Trimberger, 1948), as the STE period was less frequently expressed (Hurnik et al., 1975; Sveberg et al., 2011) and the period of frequent SEC (Kerbrat and Disenhaus, 2004) was more difficult to define (Phillips and Schofield, 1990). Roelofs et al. (2005) studied relationships between be-

Table 4. Comparison of mutual agonistic behavior (measured as counts per hour) between periods ${ }^{1}$ of estrous cycle

\begin{tabular}{|c|c|c|c|c|c|c|c|c|}
\hline Period 1 & Period $2^{2}$ & \multicolumn{3}{|c|}{ Period 1} & \multicolumn{3}{|c|}{ Period 2} & $P$-value \\
\hline Pre-MTE13-24 & Pre-MTE10-12 & 0.11 & 0.01 & 0.27 & 0.05 & 0 & 0.13 & 0.60 \\
\hline Pre-MTE10-12 & Pre-MTE7-9 & 0.05 & 0 & 0.13 & 0.13 & 0 & 0.39 & 0.72 \\
\hline Pre-MTE7-9 & Pre-MTE4-6 & 0.13 & 0 & 0.39 & 0.28 & 0.08 & 0.56 & 0.003 \\
\hline Pre-STE & STE & 1.09 & 0.48 & 2.59 & 2.69 & 1.58 & 3.96 & 0.08 \\
\hline STE & Post-STE & 2.69 & 1.58 & 3.96 & 0.70 & 0.13 & 1.40 & $<0.001$ \\
\hline Post-STE & Post-MTE1-3 & 0.70 & 0.13 & 1.40 & 0.44 & 0.08 & 0.92 & 0.55 \\
\hline Post-MTE1-3 & Post-MTE4-6 & 0.44 & 0.08 & 0.92 & 0 & 0 & 0 & 0.001 \\
\hline Post-MTE4-6 & Post-MTE7-9 & 0 & 0 & 0 & 0 & 0 & 0 & $\mathrm{NA}^{5}$ \\
\hline Post-MTE7-9 & Post-MTE10-12 & 0 & 0 & 0 & 0.10 & 0 & 0.28 & 0.23 \\
\hline Post-MTE10-12 & Post-MTE13-24 & 0.10 & 0 & 0.28 & 0.05 & 0 & 0.12 & 0.36 \\
\hline
\end{tabular}

${ }^{1}$ Period 1 and 2 of compared periods.

${ }^{2} \mathrm{NOE}=$ selected day with no cows in estrus; pre-MTE $\mathrm{x}-\mathrm{y}=$ time before mount estrus (MTE), where $\mathrm{x}$ and $\mathrm{y}$ indicate the hour interval; pre-/ post-STE = before/after standing estrus (STE); post-MTE $\mathrm{x}-\mathrm{y}=$ time after mount estrus (MTE), where $\mathrm{x}$ and $\mathrm{y}$ indicate the hour interval.

${ }^{3} P$-value for the test of difference between period 1 and period 2 .

${ }^{4}$ Lower/Upper $=$ lower/upper limit of $95 \%$ CI of period.

${ }^{5} \mathrm{NA}=$ not available. 
havioral signs and time of ovulation, and identified that the start of MTE and STE were both associated with ovulation. Behavioral changes around STE have been reported previously (Kerbrat and Disenhaus, 2004; Sveberg et al., 2011). We present results during MTE divided into 3 consecutive phases named, respectively, prestand, STE and poststand, to address differences between phases of estrus. The suggested phases of MTE are based on the original definitions (proestrus, true estrus, and metestrus) of Hurnik et al. (1975). The behavioral changes of prestand, STE, and poststand periods identified in the present report might be associated with time of ovulation, and their use could simplify decisions about when to breed.

In the present definition of SAG 3-5, all SEC are included, not only standing and mounting behaviors that define SAG in previous reports (Kilgour et al., 1977; Law et al., 2009). Defining SAG by observing standing and mounting behaviors in previous studies (Kilgour et al.,1977; Ramírez-Iglesia et al., 2006; Law et al., 2009) would have excluded estrous cows in SAG not expressing these behaviors, due to the reported decline in standing and mounting behaviors (Dobson et al., 2008). Reports of low frequencies of standing and mounting behavior emphasize the importance of using other estrus signs (Dobson et al., 2008), even though SEC are also observed at other stages of the estrous cycle (Phillips and Schofield, 1990). An additional challenge is that mounting behaviors last only for seconds (Hurnik et al., 1975) and therefore may be difficult to detect. To avoid the error of including cows randomly expressing SEC (Phillips and Schofield, 1990), a minimum frequency of SEC was mandatory in our definition of SAG 3-5. Continuous proximity to a partner cow was an additional prerequisite, and SAG 3-5 could therefore more easily be detected on a farm level, compared with single estrus signs of short duration. The prerequisite maximum distance was defined to be less than the preferred social distance of cows (Kondo et al., 2003).

\section{Duration of Estrus and Estrus Behaviors}

The reported prevalent SAG 3-5 behaviors support the view that a preference to stay in the vicinity of other cows is associated with estrus (Beach, 1976) and that proximity between cows involved in estrus behavior for a period can be used directly as an estrus observation method. To our knowledge, an estrus sign based on proximity between cows of such long duration has not previously been reported. This implies that mechanisms other than single behaviors are associated with estrus in cattle. As an indicator of estrus, SAG 3-5 was of very long duration compared with the short duration of the more frequently used sign of mounting (Hurnik et al., 1975). The total duration of SAG 3-5 was 1.45 h compared with the total duration of mounts of 38 s during MTE. To our understanding, no other sign has been described with such vast distribution through estrus, and SAG 3-5 may therefore be easier to detect at the farm level than the traditional signs with shorter duration.

\section{Sexually Active Group Fraction}

The SAG 3-5 behavior reported in the present study was confirmed to be associated with estrus and was distributed through the estrous cycle in a pattern similar to SEC behaviors. In contrast to SAG behavior described in previous studies (Law et al., 2009), SAG 3-5 was not only associated with cows in STE or MTE. During the pre- and post-estrus periods 4 to $24 \mathrm{~h}$ before MTE and 4 to $24 \mathrm{~h}$ after MTE, no SAG 3-5 was observed. The increase in SAG 3-5 during the last $3 \mathrm{~h}$ before MTE and the strong decrease during the poststand period is in agreement with previous studies reporting that cows join the SAG in pre-estrus (Kilgour et al., 1977) before their first mount and depart the group after the last mount is received (Pennington et al., 1986).

\section{Effect of Number of Cows in Standing Estrus}

The high prevalence during STE and the absence of SAG 3-5 on the day when no cows were in STE are in agreement with previous reports of estrous cows joined in groups (Ramírez-Iglesia et al., 2006; Law et al., 2009). Also, SAG 3-5 was observed during periods when a single cow was in estrus. These observations contrast with the report of Williamson et al. (1972) describing formation of SAG during synchronized breeding, when multiple cows were in estrus at a single time. Nevertheless, SAG 3-5 was 3.6 times more prevalent when 2 cows were in STE simultaneously, compared with cows alone in STE. This is in agreement with effects of number of cows in estrus on mounting behavior (Roelofs et al., 2005; Sveberg et al., 2011) and chin resting (Sveberg et al., 2011). During periods when at least 1 cow was in STE, the estrous cow participated in all SAG 3-5. This suggests that one cow in estrus is sufficient for the formation of SAG 3-5. Our results also show that SAG 3-5 can occur when there is only a single estrous cow, and that its distribution through the estrous cycle is similar to other behaviors, in contrast with the reports of other authors (Ramírez-Iglesia et al., 2006; Law et al., 2009). These differences were largely due to different definitions of SAG between studies. We suggest that SAG 3-5 is a sign of estrous cows in general that is also expressed by cows alone in estrus and cows not expressing mounting or standing behavior. 


\section{Nonestrous Cows}

Cows in the luteal phase have been described as expressing sporadic mounting behavior and SEC (Sveberg et al., 2011). In the present study, they joined SAG 3-5 only when 1 cow was alone in MTE. Cows in different phases of the estrous cycle joined the cow that was alone in estrus in her SAG 3-5, but cows close to MTE were more frequent partners. This indicates a preference for other estrous cows as SAG 3-5 partners, in agreement with previous studies (Ramírez-Iglesia et al., 2006), but confirms that any cow may join a cow alone in estrus.

\section{Secondary Estrus Signs}

The distribution of SAG 3-5 resembled the distribution of SEC initiated through the pre-estrus and prestand periods, but increased more distinctly, similar to the increase in receptive behavior, during STE. Hurnik et al. (1975) suggested from their data that more cows mounted, compared with cows mounting, were in estrus, supporting the importance of receptive behavior associated with true estrus (STE). Estrus signs are reported to increase in the hours immediately before and during estrus (Kerbrat and Disenhaus, 2004), in agreement with the results of SAG 3-5, SEC, and agonistic behaviors in the present study. However, these results do not support the observations reported by Hurnik (1987) that signs of estrus increase during the last 24 to $48 \mathrm{~h}$ before estrus. In the poststand and post-MTE periods, SAG 3-5 decreased to a greater extent than SEC, both initiated and received.

\section{Agonistic Behavior}

Mutual behaviors (head butt and play rub) followed similar patterns as SAG 3-5 and SEC throughout the estrous cycle in the present study, supporting that these signs should be regarded as estrus signs (Sveberg et al., 2011). Other agonistic behaviors differed from this pattern, with high frequencies observed during nonestrus and lower frequencies observed 13 to $24 \mathrm{~h}$ before MTE. This may be explained by pre-estrous cows being less prone to agonistic behaviors and more interested in socializing when approaching estrus. This is in agreement with the observed gradual increase in SEC initiated during the last $24 \mathrm{~h}$ before MTE and the increase in mutual agonistic behaviors during the $9 \mathrm{~h}$ before MTE. The decrease in agonistic interactions could serve as a pre-estrus sign and a warning of estrus to come. This should be further investigated because documentation of pre-estrus signs is limited and social behaviors other than the primary and secondary estrus signs are reported in few other studies (Hurnik et al., 1975; Phillips and Schofield, 1990; Kerbrat and Disenhaus, 2004). Agonistic behaviors were prominent during estrus and observation of these signs should be included in future estrus detection programs.

The difference between agonistic social behaviors initiated and received that was observed during STE has not, to our knowledge, been reported previously. The results reported here are in contrast to those of Hurnik et al. (1975), who found differences only on the days before and after estrus. They are, however, in agreement with Kerbrat and Disenhaus (2004), who reported a significant increase in agonistic behaviors initiated during the day of estrus. Those authors also observed more receptive than proceptive behaviors the day after estrus than in the present study but did not analyze these behaviors further. We found no difference between SEC initiated and received during STE. Differences between SEC initiated versus received were detected during the last hours before STE (1 to $6 \mathrm{~h}$ before MTE and prestand), but the greatest receptivity was associated with STE, for both SEC and agonistic behaviors. These results support the characterization of MTE as the proceptive period and STE as the receptive period (Hurnik, 1987).

\section{Differences Between SAG and SEC}

Analysis of the differences between SAG 3-5 and SEC in the present study enables comparisons of variables with different scales (fraction and frequencies, respectively). Initiated SEC increased slightly more than SAG 3-5 during the last $6 \mathrm{~h}$ before MTE, but the 2 variables changed similarly during prestand. The increase in SAG 3-5 was greater than that of SEC received the last $6 \mathrm{~h}$ before MTE. This indicates that increased SEC initiated and SAG 3-5 are indicators of late pre-estrus at a time when low frequencies of receptive behaviors are probably not detectable. During STE, the fraction of time spent in SAG 3-5 increased more than the frequency of initiated SEC, but was similar to the frequency of received SEC. This implies that SAG 3-5 follows a similar pattern as the previously reported receptive behaviors (Sveberg et al., 2011) during STE and that they are strongly associated with this important stage of estrus. During poststand, the fraction of time spent in SAG 3-5 decreased more than the frequency of SEC either initiated or received. A sudden decrease in the fraction of time in SAG 3-5 may more precisely signal that a cow is about to end STE than do reductions in the frequencies of other signs of estrus during the poststand phase.

High variability of estrus expression among individuals (Diskin and Sreenan, 2000) makes it difficult to ob- 
tain conclusive evidence from behavioral studies with few animals, such as the present one. Facility design, space allowances (Kondo et al., 2003; Palmer et al., 2012), and the quality of underfoot surfaces (Britt et al., 1986) may also have affected cow interactions and expression of behaviors in the present study. Nevertheless, continuous behavior studies, such as the present one, are important if novel behavior patterns that can be exploited to improve cow management are to be revealed (Williamson et al., 1972). Information about group dynamics can serve as an important and novel link between estrus behavior and other social interactions in cattle. Other characteristics of sexual and social behaviors may still be undiscovered. Further studies of SAG 3-5 and social behaviors and their relation to specific stages of estrus need to be carried out within systems in common use in dairy production.

\section{CONCLUSIONS}

The current study presents the novel measure SAG 3-5, identified by proximity to other cows and intensity of estrus behavior, as a means to quantify the SAG within a herd of cows. Mutual social agonistic behaviors, SAG 3-5, and SEC were expressed in similar patterns through estrous cycle. Although SAG 3-5 was the sign of estrus of longest duration, it was not confined only to groups of estrous cows. Reduced agonistic interactions indicated early pre-estrus. Increased SEC initiated and SAG 3-5 indicated late pre-estrus and early estrus (prestand). High frequencies of SAG 3-5, SEC, and social agonistic behaviors were indicators of STE. A decrease in SAG 3-5, preceded by a peak in interactions, was an indicator of late estrus (poststand). The use of SAG 3-5, in combination with agonistic behaviors and SEC, could improve estrus detection rates in dairy cattle.

\section{ACKNOWLEDGMENTS}

The authors gratefully acknowledge Noel Byrne and the staff at Ballydague farm (Teagasc, Moorepark, Fermoy, Ireland) for their care of the animals and help with this study. We thank Jostein Rudi and Timotei Data (Ringebu, Norway), for technical support with video recording; Ellen Dahl at the Norwegian School of Veterinary Science (Oslo) for laboratory assistance; Jörund Hage at the National Institute of Nutrition and Seafood Research (Bergen, Norway) for help with data handling; and Joan Cooper and Gary Rogers of Geno Global (Hamar, Norway) for language improvements. Support for the preparation of the antiprogesterone monoclonal antibody and the progesterone peroxidase conjugate was provided by the Estonian Science
Foundation (Tallinn, Estonia). The study was funded by the Norwegian Research Council projects "Oestrus and Oestrus Behaviour in NRF and Holstein Breeds in Modern Cattle Housing Systems" (173974/I10).

\section{REFERENCES}

Albright, J. L., and C. W. Arave. 1997. Reproductive behaviour. Pages 82-89 in The Behaviour of Cattle. CAB International, New York, NY.

Beach, F. A. 1976. Sexual attractivity, proceptivity, and receptivity in female mammals. Horm. Behav. 7:105-138.

Britt, J. H., R. G. Scott, J. D. Armstrong, and M. D. Whitacre. 1986. Determinants of estrous behavior in lactating Holstein cows. J. Dairy Sci. 69:2195-2202.

Coleman, D. A. 1993. Detecting estrus in dairy cattle. Alabama Cooperative Extension Service. Auburn Univ., AL. Accessed Oct. 20, 2012. http://www.aces.edu/pubs/docs/A/ANR-0496/ANR-0496. pdf.

Diskin, M. G., and J. M. Sreenan. 2000. Expression and detection of oestrous in cattle. Reprod. Nutr. Dev. 40:481-491.

Dobson, H., S. L. Walker, M. J. Morris, J. E. Routly, and R. F. Smith. 2008. Why is it getting more difficult to successfully artificially inseminate dairy cows? Animal 2:1104-1111.

Efron, B., and R. Tibshirani. 1993. An Introduction to the Bootstrap. Chapman \& Hall/CRC, Boca Raton, FL.

Galina, C. S., A. Orihuela, and I. Rubio. 1996. Behavioural trends affecting oestrus detection in Zebu cattle. Anim. Reprod. Sci. 42:465-470.

Hafez, E. S. E., and M. F. Bouissou. 1975. The behaviour of cattle. Pages 203-245 in The Behaviour of Domestic Animals. 3rd ed. Williams and Wilkins, Baltimore, MD.

Hafez, E. S. E., and D. R. Lindsay. 1965. Behavioural responses in farm animals and their relevance to research technique. A review. Anim. Breed. Abstr. 33:1-16.

Hurnik, J. F. 1987. Sexual behaviour of female domestic mammals. Vet. Clin. North Am. 3:423-461.

Hurnik, J. F., G. J. King, and H. A. Robertson. 1975. Estrous and related behavior in postpartum Holstein cows. Appl. Anim. Ethol. $2: 55-68$.

Kerbrat, S., and C. Disenhaus. 2004. A proposition for an updated behavioral characterisation of the oestrus period in dairy cows. Appl. Anim. Behav. Sci. 87:223-238.

Kilgour, R., B. H. Skarscholt, J. F. Smith, K. J. Bremner, and M. C. L. Morrison. 1977. Observation of the behaviour and factors influencing the sexually active group in cattle. Proc. N.Z. Soc. Anim. Prod. 37:128-135.

Kondo, S., J. Sekine, M. Okubo, and Y. Asahida. 2003. The effect of group size and space allowance on the agonistic and spacing behavior of cattle. Appl. Anim. Behav. Sci. 24:127-135.

Law, R. A., F. J. Young, D. C. Patterson, D. J. Kilpatrick, A. R. G. Wylie, and C. S. Mayne. 2009. Effect of dietary protein content on estrous behavior of dairy cows during early and mid lactation. J. Dairy Sci. 92:1013-1022.

Lowman, B. G., N. A. Scott, and S. H. Somerville. 1976. Condition Scoring of Cattle. Rev. ed. Bulletin no. 6. East of Scotland College of Agriculture, Edinburgh, UK.

Mülleder, C., P. Palme, C. Menke, and S. Waiblinger. 2003. Individual differences in behavior and in adrenocortical activity in beef-suckler cows. Appl. Anim. Behav. Sci. 84:167-183.

O'Driscoll, K., A. Hanlon, and L. Boyle. 2008. The effect of out-wintering pad design on the synchrony of dairy cow behavior. J. Dairy Sci. 91:4651-4660.

Orihuela, A. 2000. Some factors affecting the behavioural manifestation of oestrus in cattle: A review. Appl. Anim. Behav. Sci. $70: 1-16$

Palmer, M. A., G. Olmos, L. A. Boyle, and J. F. Mee. 2012. A comparison of the estrous behavior of Holstein-Friesian cows when cubicle-housed and at pasture. Theriogenology 77:382-388. 
Pennington, J. A., J. L. Albright, and C. J. Callahan. 1986. Relationships of sexual activities in estrous cows to different frequencies of observation and pedometer measurements. J. Dairy Sci. 69:2925-2934.

Phillips, C. J. C., and S. A. Schofield. 1990. The effect of environment and stage of the oestrous cycle on the behaviour of dairy cows. Appl. Anim. Behav. Sci. 27:21-31.

Ramírez-Iglesia, L. N., F. B. Viera Rosales, J. A. Martinez, A. D. de Ramírez, J. G. Morillo Luque, R. Román, and E. Soto-Belloso. 2006. Sexually active groups in dual purpose postpartum crossbred cattle. Zootec. Trop. 24:281-295.

Roelofs, J. B., F. J. van Eerdenburg, N. M. Soede, and B. Kemp. 2005. Various behavioral signs of estrous and their relationship with time of ovulation in dairy cattle. Theriogenology 63:1366-1377.

Sprecher, D. J., D. E. Hostetler, and J. B. Kaneene. 1997. A lameness scoring system that uses posture and gait to predict dairy cattle reproductive performance. Theriogenology 47:1179-1187.

Sveberg, G., A. O. R. Refsdal, H. W. Erhard, E. Kommisrud, M. Aldrin, I. F. Tvete, F. Buckley, A. Waldmann, and E. Ropstad. 2011. Behavior of lactating Holstein-Friesian cows during spontaneous cycles of estrus. J. Dairy Sci. 94:1289-1301.
Sveberg, G., L. E. Ruud, J. Sunde, and O. Østerås. 2007. Mål på NRF kua. (The size of the Norwegian Red Dairy cow). Geno report no. 1/ 07. Geno, Hamar, Norway.

Trimberger, W. T. 1948. Breeding efficiency in dairy cattle from artificial insemination at various intervals before and after ovulation. Univ. Nebraska Agric. Exp. Station Res. Bull. 153:3-25.

Van Vliet, J. H., and F. J. C. M. van Eerdenburg. 1996. Sexual activities and oestrus detection in lactating Holstein cows. Appl. Anim. Behav. Sci. 50:57-69.

Waldmann, A. 1993. Enzyme immunoassay (EIA) for milk progesterone using a monoclonal antibody. Anim. Reprod. Sci. 34:19-30.

Waldmann, A. 1999. Monoclonal antibodies to progesterone: Characterization and selection for enzyme immunoassay in bovine milk. Hybridoma 18:289-296.

Williamson, N. B., R. S. Morris, D. C. Blood, and C. M. Cannon. 1972. A study of oestrous behaviour and oestrus detection methods in a large commercial dairy herd. I. The relative efficiency of methods of oestrus detection. Vet. Rec. 91:50-58. 